\title{
sciendo
}

Int. J. of Applied Mechanics and Engineering, 2018, vol.23, No.3, pp.595-609

DOI: 10.2478/ijame-2018-0032

\section{NATURAL CONVECTION IN A HYDRODYNAMICALLY AND THERMALLY ANISOTROPIC NON-RECTANGULAR POROUS CAVITY: EFFECT OF INTERNAL HEAT GENERATION/ABSORPTION}

\author{
P. CHANDRAN ${ }^{*}$ and N.C. SACHETI \\ Department of Mathematics and Statistics, College of Science \\ Sultan Qaboos University, PC 123, Al Khod, Muscat, SULTANATE OF OMAN \\ E-mails: chandran@squ.edu.om; nirmal@squ.edu.om \\ B.S. BHADAURIA \\ Department of Mathematics, School of Physical Sciences \\ Babasaheb Bhimrao Ambedkar University \\ Lucknow 226025, INDIA \\ Department of Mathematics, Banaras Hindu University, Varanasi 221005, INDIA \\ E-mail: mathsbsb@yahoo.com
}

\author{
A.K. SINGH \\ Department of Mathematics, Banaras Hindu University \\ Varanasi 221005, INDIA \\ E-mail: ashok@bhu.ac.in
}

\begin{abstract}
Laminar natural convection in a trapezoidal porous vertical cavity has been investigated in this work. It is assumed that the porous enclosure is filled up with a permeable material subject to hydrodynamic and thermal anisotropy, the flow being governed by the Darcy law as applicable to a non-isotropic medium. It is further assumed that (i) there is heating at the left vertical wall and cooling at the right wall of the enclosure and (ii) the flow domain is subject to the presence of heat source or heat sink. The partial differential equations governing the resulting free convection have been solved numerically in the non-dimensional forms. There arises a number of parameters relating to buoyancy, internal heating, cavity aspect ratio and inclination of the upper surface to the horizontal. The influence of these parameters has been illustrated and analyzed through contours of streamlines and isotherms. We have also discussed the role of internal heating as well as anisotropy on the heat transfer characteristics.
\end{abstract}

Key words: natural convection, trapezoidal cavity, porous medium, permeability, thermal diffusivity, Darcy law, heat source/sink.

2010 Mathematics Subject Classification. 76R10, 76S05, 76D99

\section{Introduction}

The anisotropy features in the theoretical analysis of flow and heat transfer in porous media of finite or infinite extent have received considerable attention in the literature on porous media flows. In particular, a large number of researchers have focused their attention on investigating natural convection in incompressible viscous fluids within the confines of finite permeable enclosures because of a wide range of

\footnotetext{
${ }^{*}$ To whom correspondence should be addressed
} 
real life applications in engineering and industries. For instance, the applications such as storage of energy, storage of nuclear waste material, production of geothermal energy from reservoirs, oil extractions, thermal insulation, heating or cooling of buildings, to name a few, all require deep mathematical analysis of the momentum and energy transport equations governing free convective flows in a variety of geometrical configurations and subject to a range of physical conditions.

In the investigations of two-dimensional natural convective flows in finite enclosures in a variety of geometrical shapes such as squares, rectangles, trapezoids, triangles, filled with anisotropic porous material, the orientation of the principal axes is known to play a pivotal role, for example, in assessing maximum or minimum heat transfer rates. In a number of studies relating to natural convection in finite permeable enclosures, one of the principal axes is assumed to be either in the direction of gravity or some specific direction (i.e., oblique principal axes) depending upon the nature of applications. There is another key feature worth mentioning with regard to the type of anisotropy. A close look at the literature apparently reveals that three types of anisotropic features have generally been accounted for while discussing free convective flow: (i) anisotropy due to permeability variations only; (ii) anisotropy due to changes in thermal diffusivity only and (iii) anisotropy due to variations in both quantities. The effect of one or more of such features of anisotropy has been amply exhibited on isotherms, streamlines and a few important quantities of engineering interest, e.g., skin friction and heat transfer coefficients at a bounding surface for a variety of thermal conditions at the enclosure walls, namely, isothermal, adiabatic and constant heat flux in a large number of investigations in literature. Both Darcy as well as non-Darcy models for flows in a porous medium were employed in such studies. On the other hand, a number of investigations on stability analyses relating to the onset of convection in porous media of finite thickness or finite lateral extent have also considered various anisotropic features. For references on all aforementioned aspects of anisotropic properties in free convective flows, one may refer to works reported in [1-16].

In the study of buoyancy dominated convective flows of viscous incompressible fluids occupying finite or semi-infinite porous regions, there is yet another key aspect which has drawn the attention of researchers in recent years. This relates to the effect of internal heat generation due to the presence of heat sources or sinks in the flow domain. A number of studies in the literature [17-23] have been reported dealing with this particular feature. The present paper is devoted to studying numerically free convection in a vertical non-rectangular anisotropic region in the shape of a trapezoid, with a sloping upper boundary. It is assumed that the region has the presence of sources/sinks. We are thus extending an earlier work [15] with an aim to analyze the effect of internal heat source/sink. The effect of each of a number of governing non-dimensional parameters, namely, the Rayleigh number, internal heat source/sink parameter, aspect ratio and inclination angle of upper surface, assuming the remaining parameters to be fixed, has been exhibited on isotherms and streamlines of the flow. We have also computed and analyzed the average Nusselt number at a bounding surface by fixing the Rayleigh number, inclination angle and the aspect ratio while varying others including the thermal diffusivity parameter.

\section{Mathematical formulation}

We consider natural convection in a non-rectangular cavity, in the shape of a finite trapezoid, whose side walls are vertical. The basic dimensions of the cavity are $L$ (width) and $H$ (height). We assume that, with respect to a two-dimensional coordinate system $O x y$, the $y$-axis is along the left vertical wall while the $x$-axis is normal to it into the fluid, as indicated in Fig.1. We further assume that (a) the cavity is filled up with a permeable material subject to hydrodynamic and thermal anisotropy, and saturated with an incompressible viscous fluid, (b) the side walls are kept at uniform but different temperatures $T_{1}$ and $T_{2}$, respectively, $\left(T_{1}>T_{2}\right)$, (c) the lower horizontal and upper slanted surfaces are adiabatic, and (d) the flow domain is subject to the presence of heat generating or absorbing sources or sinks. The temperature gradient arising due to differential in side surface temperatures leads to free convection currents within the cavity space. Further, the Boussinesq approximation, widely used in the literature for natural convective flows in 
both bounded and unbounded domains, will be employed allowing the density $\rho$ of the fluid to be expressed as $\rho=\rho_{0}\left[1-\beta\left(T-T_{1}\right)\right]$, where $\beta$ is the coefficient of thermal expansion and $T$ is the fluid temperature.

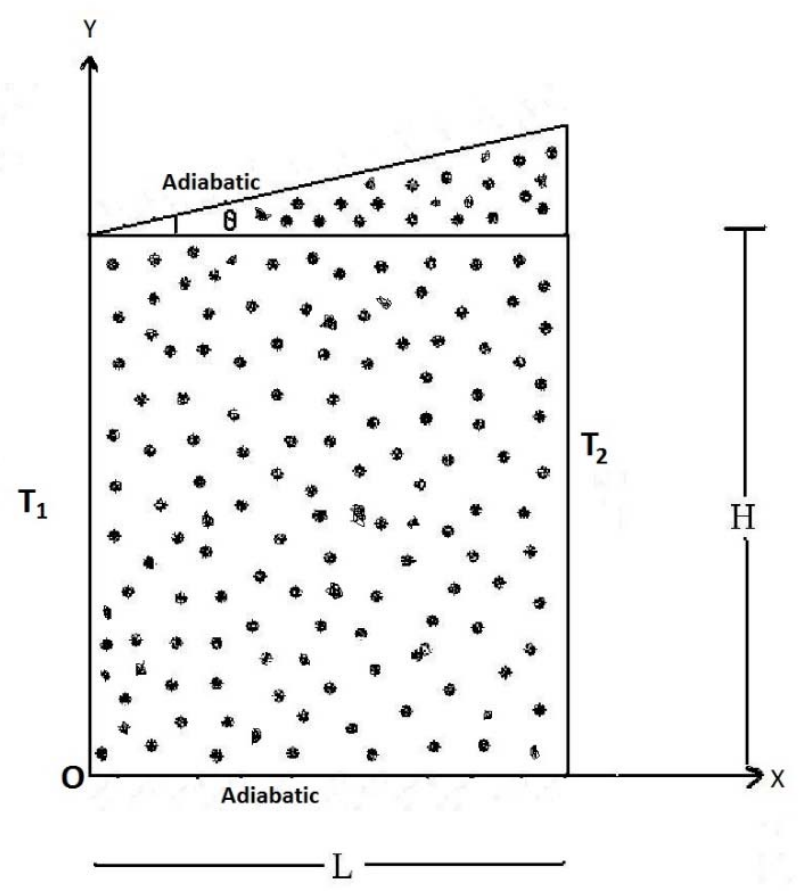

Fig.1. Physical configuration.

In view of the above geometrical and physical considerations, and assuming the flow in the porous enclosure to be modeled by the Darcy law for a non-isotropic medium, the partial differential equations governing such a free convective flow are a modified version of an earlier study [15], and are given by

$$
\begin{aligned}
& \nabla \cdot \boldsymbol{V}=0, \\
& \mu \boldsymbol{V}+K_{x y}(\nabla p+\rho g \boldsymbol{j})=0, \\
& (\boldsymbol{V} \cdot \nabla) T-\nabla \cdot\left(\alpha_{x y} \nabla T\right)=Q T
\end{aligned}
$$

where $\boldsymbol{V}$ is the flow velocity, $\mu$ the viscosity, $p$ the pressure, $g$ the gravitational acceleration, $Q$ is the heat source/sink coefficient, and

$$
K_{x y}=\left[\begin{array}{cc}
K_{x} & 0 \\
0 & K_{y}
\end{array}\right], \quad \alpha_{x y}=\left[\begin{array}{cc}
\alpha_{x} & 0 \\
0 & \alpha_{y}
\end{array}\right] .
$$

In Eq.(2.4), the tensorial quantities $K_{x y}$ and $\alpha_{x y}$ represent, respectively, anisotropy in permeability and thermal diffusivity, assuming the principal axes are along the coordinate axes.

We now introduce the following non-dimensional quantities

$$
(\tilde{x}, \tilde{y})=(x / H, y / H), \quad(\tilde{u}, \tilde{v})=\left(H u / \alpha_{y}, H v / \alpha_{y}\right)
$$




$$
\begin{aligned}
& \tilde{T}=\frac{T-T_{2}}{T_{1}-T_{2}}, \quad K=K_{x} / K_{y}, \\
& \alpha=\alpha_{x} / \alpha_{y}, \quad \mathrm{Da}=K_{x} / H^{2}, \\
& \mathrm{Ra}=\frac{\rho g \beta H^{3}\left(T_{1}-T_{2}\right)}{\mu \alpha_{y}}, \quad \Lambda=(\mathrm{Da})(\mathrm{Ra}), \\
& A r=L / H, \quad \tilde{Q}=H^{2} Q / \alpha_{y} .
\end{aligned}
$$

The non-dimensional parameters occurring in the above equation are: permeability and thermal diffusivity ratios $K, \alpha$, the Darcy number Da, Rayleigh number Ra, aspect ratio $A r$, heat source/sink parameter $\tilde{Q}$, and Rayleigh-Darcy number $\Lambda$.

Introducing the non-dimensional stream function $\Psi$ such that $u=\partial \Psi / \partial y, \quad v=-\partial \Psi / \partial x$, the continuity equation is automatically satisfied. Furthermore, Eqs (2.2) and (2.3) assume the forms (neglecting the "tilde" on the quantities, for convenience)

$$
\begin{aligned}
& K \frac{\partial^{2} \Psi}{\partial x^{2}}+\frac{\partial^{2} \Psi}{\partial y^{2}}=-\Lambda \frac{\partial T}{\partial x} \\
& u \frac{\partial T}{\partial x}+v \frac{\partial T}{\partial y}=\alpha \frac{\partial^{2} T}{\partial x^{2}}+\frac{\partial^{2} T}{\partial y^{2}}+Q T
\end{aligned}
$$

In a non-dimensional form, the boundary conditions are given by

$$
\begin{aligned}
& \Psi=0 \text { on all boundaries, } \\
& T=1 \text { at } f_{1}(x, y)=0 \text { and } T=0 \text { at } f_{2}(x, y)=0, \\
& \frac{\partial T}{\partial n}=0 \text { at } f_{3}(x, y)=0 \text { and } f_{4}(x, y)=0
\end{aligned}
$$

where the functions $f_{1}, f_{2}, f_{3}$ and $f_{4}$ represent bounding surfaces of the non-rectangular domain (Fig.2), and $\boldsymbol{n}$ is the normal to the boundary.

\section{Numerical solution}

The numerical solution procedure of the boundary value problem described by Eqs (2.6)-(2.8) follows closely the method used in one of our earlier studies [15]. However, for the sake of completeness, some pertinent details are reproduced here. The solution is accomplished by an algebraic grid generation method combined with a suitable finite difference method [24-27]. For this, the physical trapezoidal domain of the problem is transformed to a unit square domain by the transformation

$$
\xi=\xi(x, y), \quad \eta=\eta(x, y)
$$


where $0 \leq \xi, \eta \leq 1$. It may be noted that one can express $\xi$ and $\eta$ explicitly in terms of $f_{1}, f_{2}, f_{3}$ and $f_{4}$, where these functions are given by

$$
f_{1}(x, y)=x, f_{2}(x, y)=x-\operatorname{Ar}, f_{3}(x, y)=y, f_{4}(x, y)=y-x \tan \theta-1 .
$$

We shall first perform the numerical computation in the unit square domain. For this, we use the transformation

$$
\odot_{x}=\odot_{\xi} \xi_{x}+\odot_{\eta} \eta_{x}, \odot_{y}=\bullet_{\xi} \xi_{y}+\square_{\eta} \eta_{y},
$$

and re-express Eqs (2.6) and (2.7) in the forms

$$
\begin{aligned}
& b_{1}(K) \frac{\partial^{2} \Psi}{\partial \xi^{2}}+b_{2}(K) \frac{\partial^{2} \Psi}{\partial \eta^{2}}+b_{3}(K) \frac{\partial^{2} \Psi}{\partial \xi \partial \eta}+b_{4}(K) \frac{\partial \Psi}{\partial \xi}+b_{5}(K) \frac{\partial \Psi}{\partial \eta}=-\Lambda\left(a_{1} \frac{\partial T}{\partial \xi}+a_{2} \frac{\partial T}{\partial \eta}\right) \\
& B_{1} \frac{\partial T}{\partial \xi}+B_{2} \frac{\partial T}{\partial \eta}=b_{1}(\alpha) \frac{\partial^{2} T}{\partial \xi^{2}}+b_{2}(\alpha) \frac{\partial^{2} T}{\partial \eta^{2}}+b_{3}(\alpha) \frac{\partial^{2} T}{\partial \xi \partial \eta}+Q T
\end{aligned}
$$

where

$$
B_{1}=a_{1} u+a_{3} v-b_{4}(\alpha), \quad B_{2}=a_{2} u+a_{4} v-b_{5}(\alpha),
$$

and the functionals $a_{1}, \cdots, a_{4}, b_{1}, \cdots, b_{4}$ are defined as

$$
\begin{aligned}
& a_{1}=\frac{\partial \xi}{\partial x}, \quad a_{2}=\frac{\partial \eta}{\partial x}, \quad a_{3}=\frac{\partial \xi}{\partial y}, \quad a_{2}=\frac{\partial \eta}{\partial y}, \\
& b_{1}(s)=s a_{1}^{2}+a_{3}^{2}, \quad b_{2}(s)=s a_{2}^{2}+a_{4}^{2}, \quad b_{3}(s)=2\left(s a_{1} a_{2}+a_{3} a_{4}\right), \\
& b_{4}(s)=2\left(s \frac{\partial^{2} \xi}{\partial x^{2}}+\frac{\partial^{2} \xi}{\partial y^{2}}\right), \quad b_{5}(s)=2\left(s \frac{\partial^{2} \eta}{\partial x^{2}}+\frac{\partial^{2} \eta}{\partial y^{2}}\right) .
\end{aligned}
$$

In Eqs (3.6), $s$ stands for either $K$ or $\alpha$. The boundary conditions applicable to the unit square domain are

$$
\begin{aligned}
& \Psi=0 \text { on all boundaries, } \\
& T=1 \text { at } \xi=0, \text { and } T=0 \text { at } \xi=1, \\
& \left(a_{1} \eta_{x}+a_{3} \eta_{y}\right) \frac{\partial T}{\partial \xi}+\left(a_{2} \eta_{x}+a_{4} \eta_{y}\right) \frac{\partial T}{\partial \eta}=0 \text { at } \eta=0 \text { and } \eta=1 .
\end{aligned}
$$



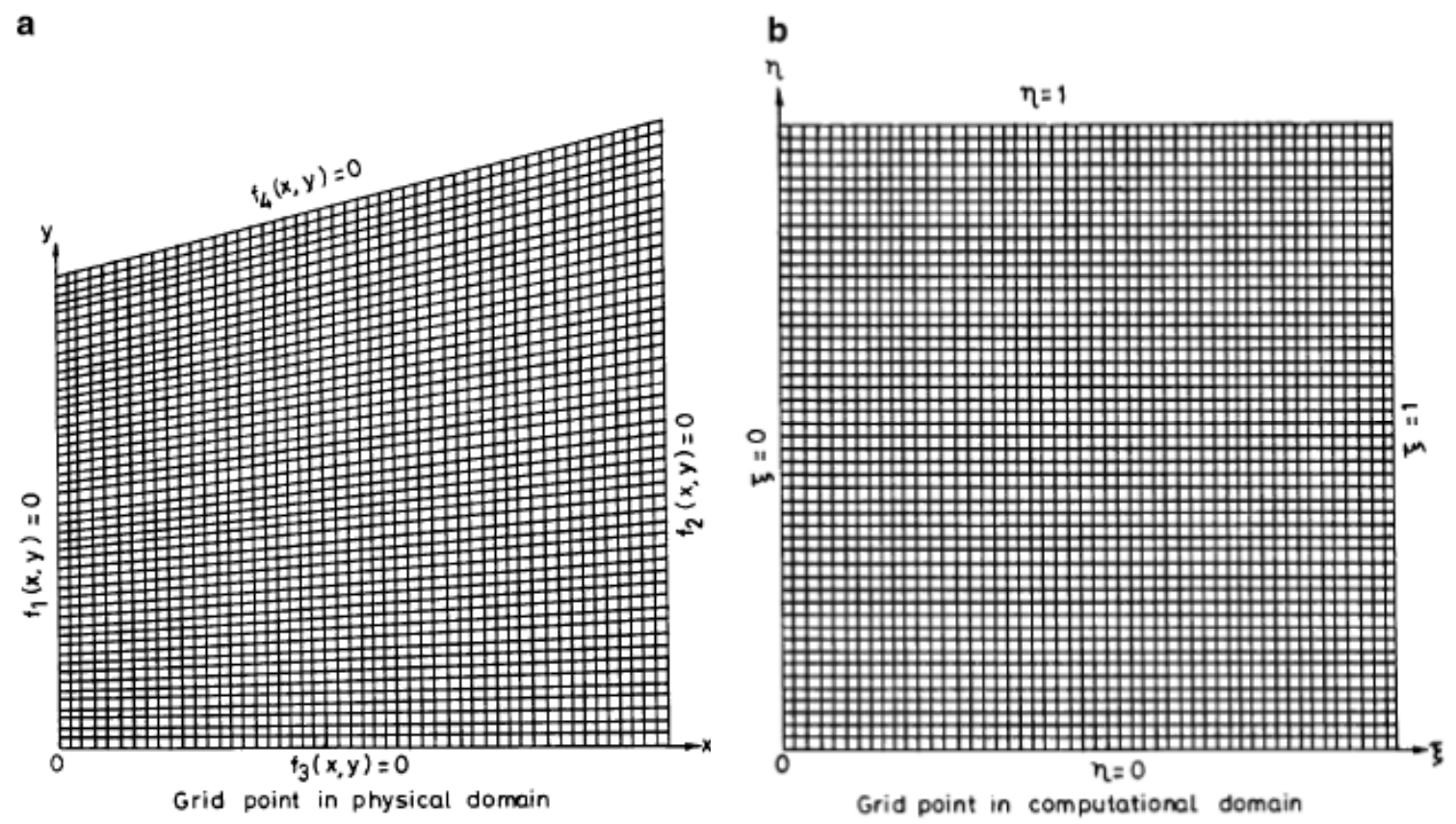

Fig.2. Physical and computational domains.

A solution of the regular boundary value problem described by Eqs (3.4), (3.5) and (3.7) can now be obtained by standard procedures [15]. The solution so obtained is valid in the unit square domain. It can then be transformed back to the physical domain using the functional relations between the physical and computational domain variables.

Nusselt number: The rate of heat transfer is estimated from the Nusselt number $\mathrm{Nu}$. The local Nusselt number $\mathrm{Nu}(y)$ at the left boundary is given by

$$
\mathrm{Nu}(y)=-\left.\frac{\partial T}{\partial x}\right|_{x=0}
$$

The overall Nusselt number $\mathrm{Nu}$ is then obtained by integrating $\mathrm{Nu}(y)$ over the left vertical boundary.

\section{Discussion of numerical results}

In this section, we shall analyze the effects of four governing parameters, namely, the internal heat generation parameter $(\mathrm{Q})$, inclination angle $(\theta)$, Rayleigh number $(\mathrm{Ra})$ and, finally, the aspect ratio $(A r)$ on the formation of streamlines and isotherms, through the set of Figs 3-10. However, we have assumed both the thermal diffusivity parameter $\alpha$ as well as the permeability parameter $K$ to be constant $(=0.5)$ in all computations relating to streamlines and isotherms.

Figures 3-5 have been included to showcase the effect of our lead parameter $Q$, keeping other parameters $\operatorname{Ar}(=2), \theta\left(=20^{\circ}\right)$, and $\mathrm{Ra}(=100)$ fixed. Here we shall include both positive and negative values of $Q$ to assess the impact of heat sources as well as sinks. In the next set of two Figs 6, 7, we shall exhibit the effect of the inclination angle from 0 to 30 degrees keeping $\operatorname{Ar}(=2), Q(=2)$, and $\mathrm{Ra}(=100)$ fixed. In Fig.8, we have demonstrated how the change in the buoyancy parameter Ra causes streamlines and isotherms to change when $\operatorname{Ar}(=2), \theta\left(=20^{\circ}\right)$, and $Q(=2)$ are kept fixed. In the last set of Figs 9 and 10, we shall 
analyze the effect of the aspect ratio when $A r$ varies from 1 to 4 while keeping $Q(=2), \theta\left(=20^{\circ}\right)$, and $\operatorname{Ra}(=$ 100) constant.

Let us now discuss in detail the variations of the contours of streamlines and isotherms with changes in the aforementioned parameters.

The streamline contours (Fig.3), as $Q$ increases from $(-5)$ to $(-1)$, show broadly similar patterns of distorted elliptic cells but with increasing boundary layer effects away from the left hot boundary. Apparently, the cells show movement towards the right boundary with an increase of $Q$ from relatively large negative values. On the other hand, as $Q$ increases from 0 to 5 (see Figs 4, 5), the streamline cells show (i) lesser boundary layer effects near all the boundaries of the enclosure except the right vertical boundary, and (ii) a change in orientation from nearly horizontal to vertical for the innermost contour indicating a different circulation pattern. A further increase in the value of $Q$ to 10 (Fig.5) brings about the most interesting features of the streamlines: formation of two distinct groups of contours, although nearly vertical now. In other words, there is a transition from a single cell formation to double cell formation in the streamlines for values of $Q$ above 5 but less than 10 .

As regards isotherms, the contours shown in the isotherms in Fig.3 correspond to the parameter $Q$ varying from $(-5)$ to $(-1)$. One can observe that the dominance of boundary layer formation near the lower part of the left hot surface shifts a fair bit to the upper part of the right cold surface with this change in $Q$. In other words, with an increase of $Q$ from high negative values to values close to zero, the region of dominance of heat transfer by conduction partly shifts to the colder region. Apart from this feature, the shape and orientation of the isotherm contours are broadly similar.
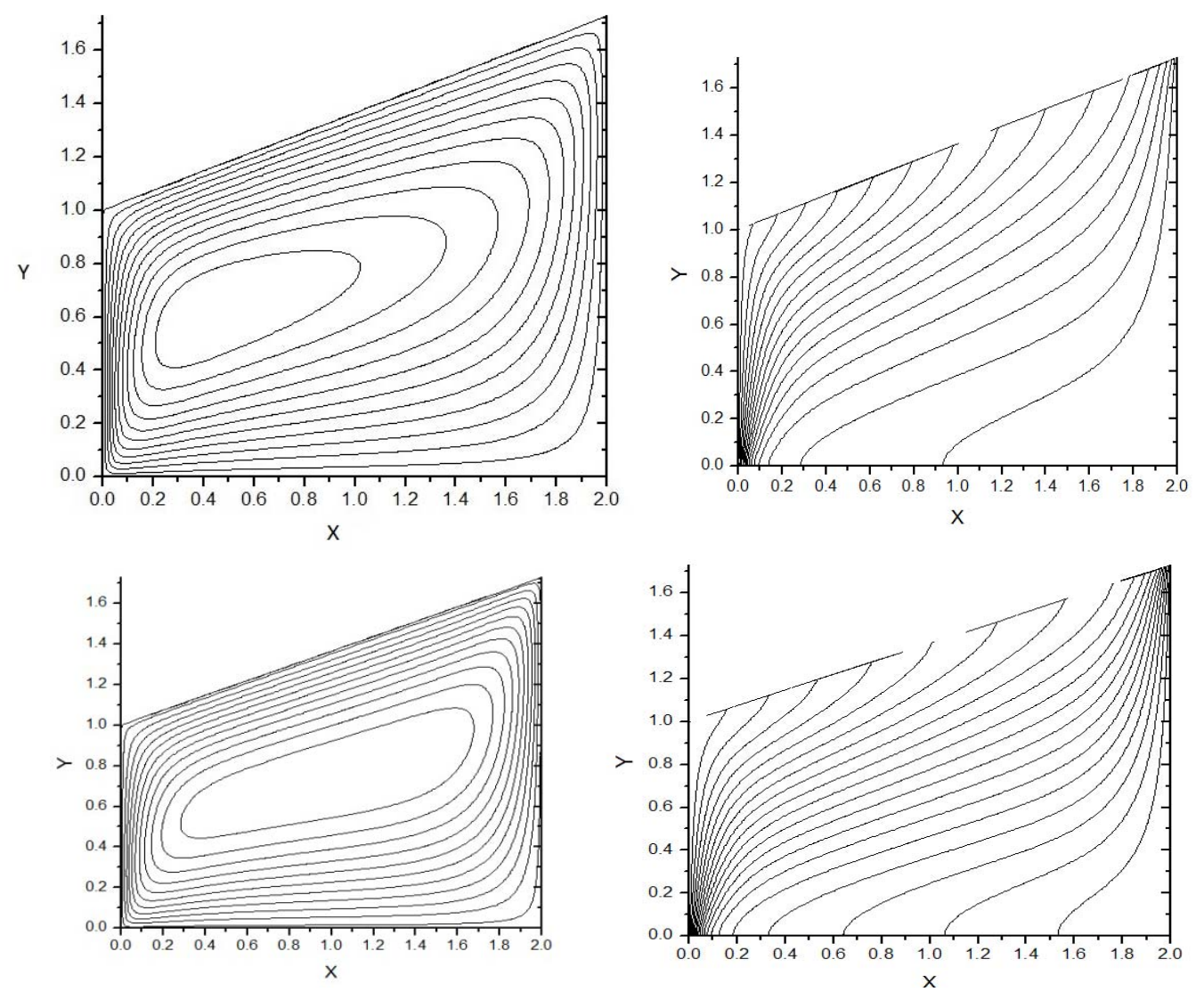

Fig.3. Streamlines (left) and isotherms (right) for $\theta=20^{\circ}, A r=2, \Lambda=100$ : Top: $Q=-5$, Bottom: $Q=-1$. 

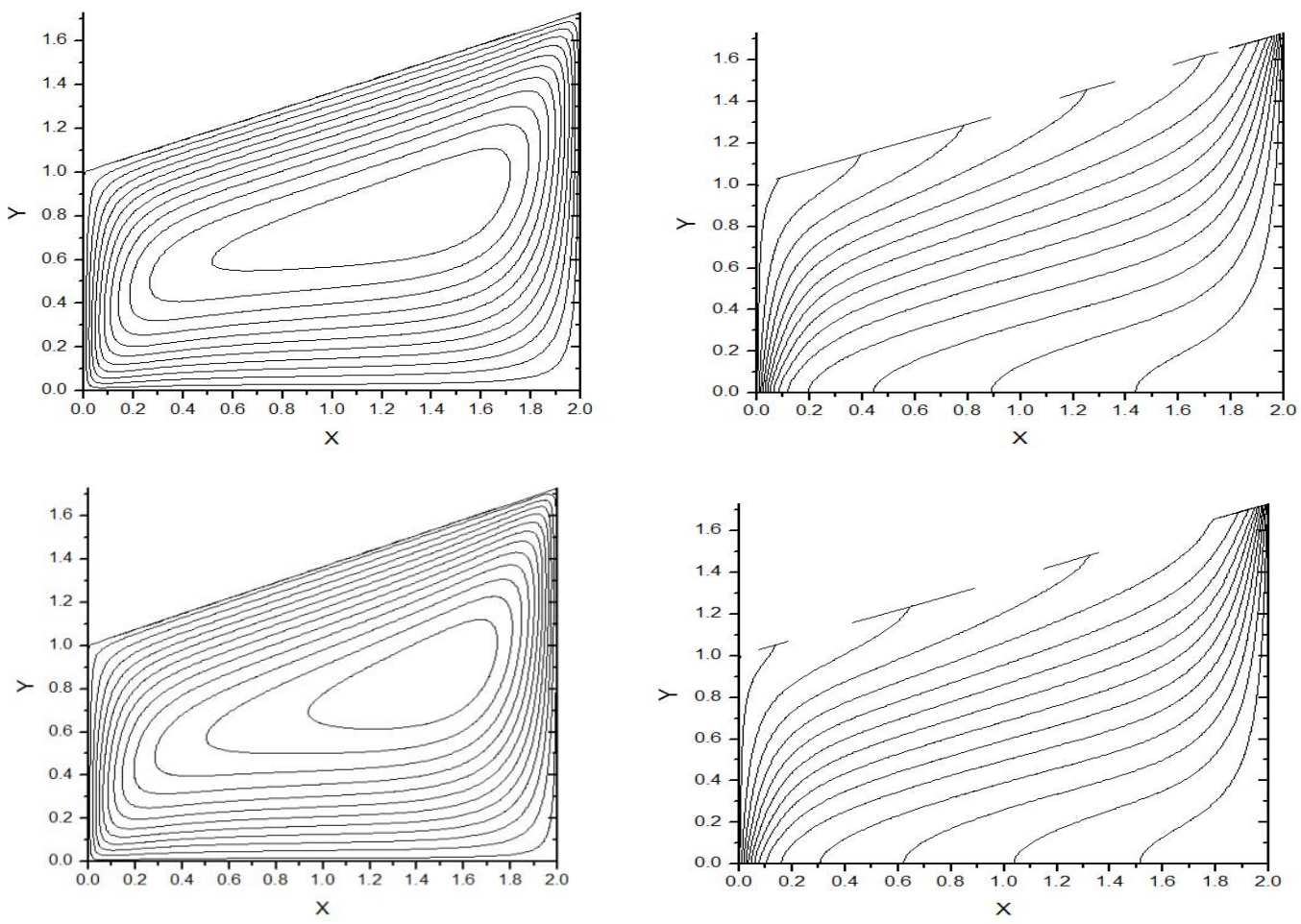

Fig.4. Streamlines (left) and isotherms (right) for $\theta=20^{\circ}, A r=2, \Lambda=100$ : Top: $Q=0$, Bottom: $Q=1$.
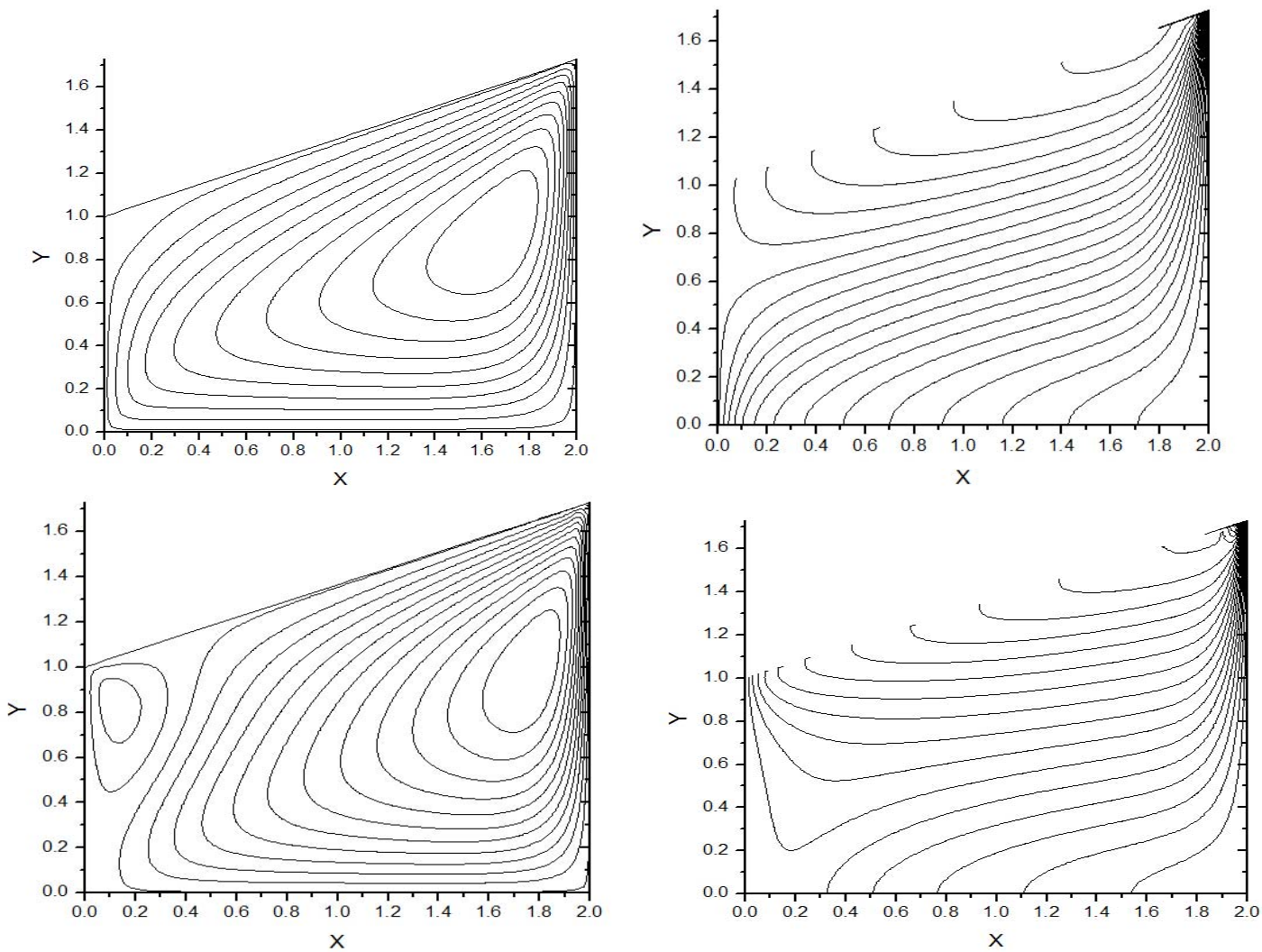

Fig.5. Streamlines (left) and isotherms (right) for $\theta=20^{\circ}, A r=2, \Lambda=100$ : Top: $Q=5$, Bottom: $Q=10$. 

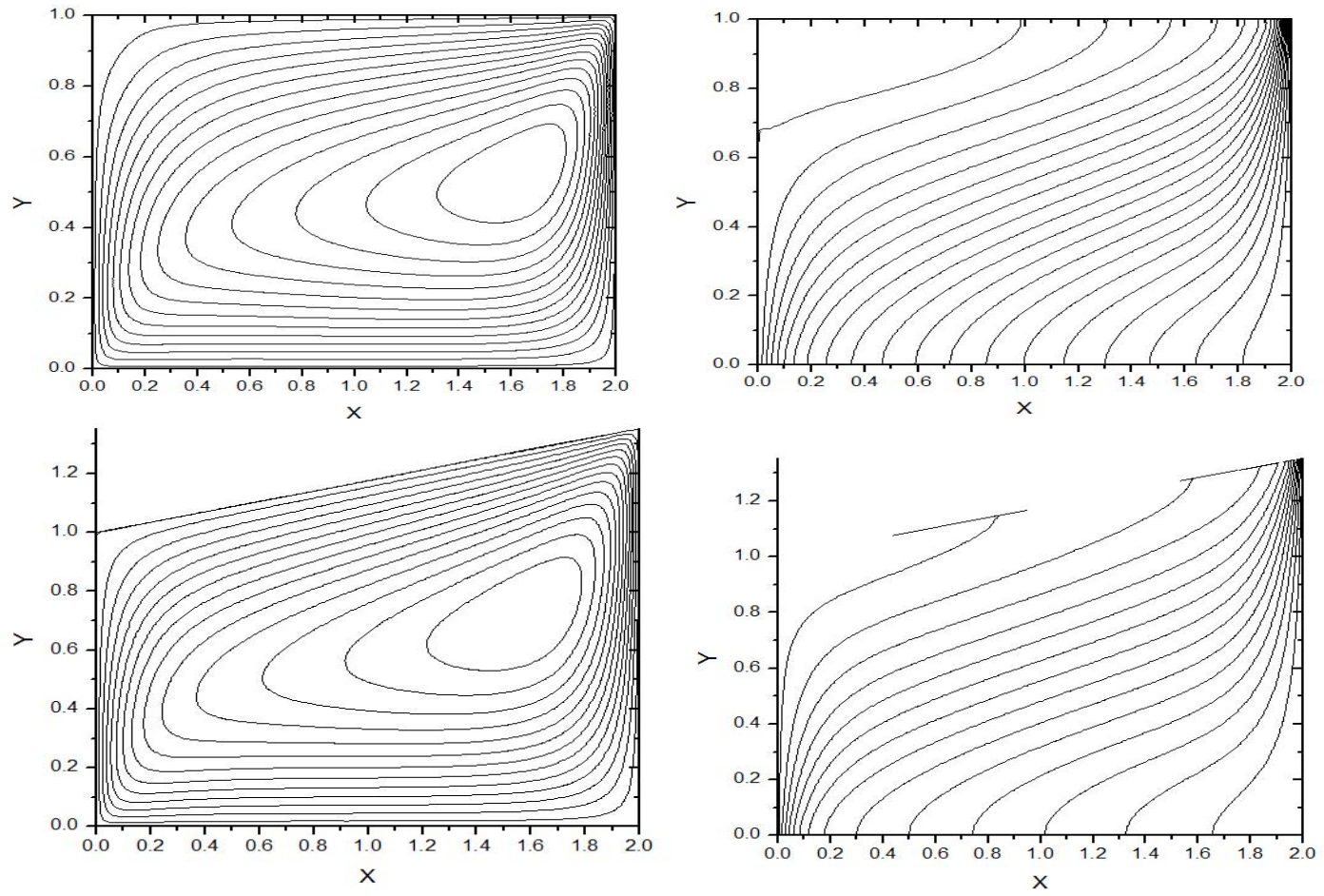

Fig.6. Streamlines (left) and isotherms (right) for $A r=2, Q=2, \Lambda=100$ : Top: $\theta=0^{\circ}$, Bottom: $\theta=10^{\circ}$.

On the examination of the set of isotherms for lower positive values, $Q=1$ and $Q=2$, versus the set of isotherms for relatively higher values, $Q=5$ and $Q=10$, one can easily notice two distinct types of isotherm formations. In the first category (Figs 4 and 5) contour curves are all seen to start from the lower (adiabatic) boundary and then reach up to the upper (adiabatic) inclined boundary. However, in contrast, the pattern of isotherm contours in the latter category (see Fig.5) shows two types of formation: either starting from the upper surface and ending up on the same surface, or starting from the lower surface and ending up on the upper inclined surface. As regards the boundary layer formation, it is easily seen that the former category contours show boundary layer formation near both the hot and cold vertical side surfaces as against the latter category contours showing apparently the boundary layer formation near the cold plate only.

As regards the influence of the upper surface inclination on the fluid convection, the results for $\theta>0$ can be used to measure how streamlines and isotherms deviate from their rectangular domain counterparts. However, a casual glance at Figs 6 and 7 appears to indicate that, overall, the effect of the inclination angle on streamlines and isotherms is not significant enough for the chosen set of values of other parameters Ra, $A r$ and $Q$. Streamlines are generally parallel to the boundaries of the enclosure except the ones (oblique but distorted ellipses) in the central region between the lower and upper surfaces, albeit closer to the cold right vertical surface. As a conjecture, it is possible that streamlines may show a fair deviation from those of the rectangular regions for some other set of values of Ra, $A r$ and $Q$. From Figs 6 and 7, one may observe that as $\theta$ increases from 0 (rectangular region) to $30^{\circ}$ (i.e., trapezoidal region), isotherms do show some distinct changes with regard to the boundary layer formation: while the boundary layer is conspicuous near the upper part of cold surface for $\theta=0^{\circ}$, the subsequent contours for higher values of $\theta$ clearly indicate conduction dominated regimes near both the hot and cold surfaces with an increase of the inclination angle. 

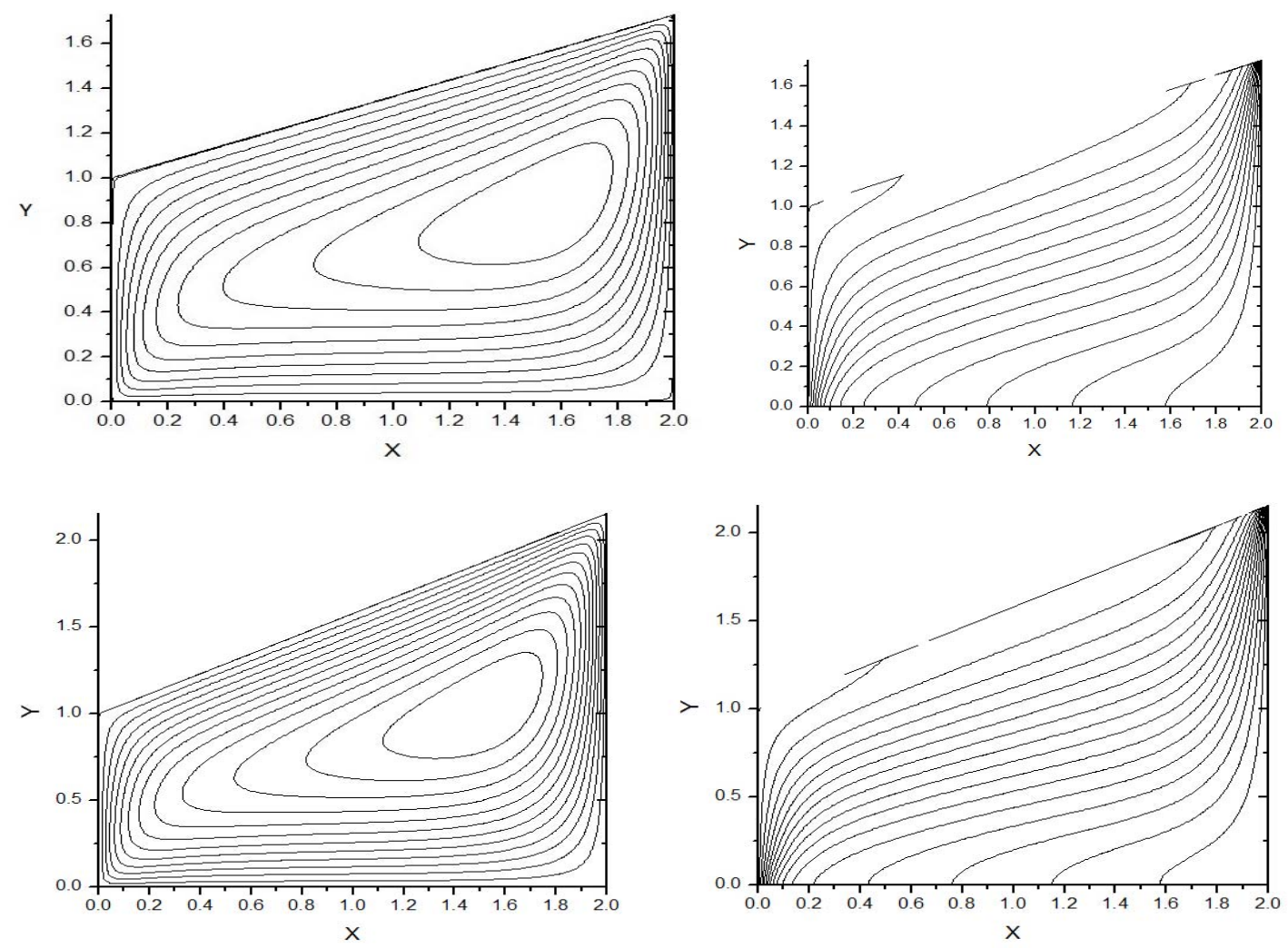

Fig.7. Streamlines (left) and isotherms (right) for $A r=2, Q=2, \Lambda=100$ : Top: $\theta=20^{\circ}$, Bottom: $\theta=30^{\circ}$.

From the contours of streamlines and isotherms shown in Fig.8, it is quite apparent that both streamlines and isotherms are sensitive to the buoyancy parameter. For instance, the streamline pattern for $\Lambda=10$ shows two distinct circulation patterns in the enclosure: one, closer to the hot wall and much less crowded, shows vertical closed loops, while the other, occupying 3/4th region of the enclosure, shows a sort of uniform pattern comprising nearly vertical curves. However, as $\Lambda$ assumes relatively large values (greater than or equal to 100) we notice only one circulation pattern. Interestingly, as $\Lambda$ assumes higher values, the boundary layer effects become quite visible on both the left and right surfaces. In the same vein, one can notice that contours of isotherms for relatively low values of $\Lambda(=10)$ are distinctly different to their counterparts for $100 \leq \Lambda$. In the former, we notice that contours are broadly parallel to either the hot or cold vertical wall, except for the region close to the central part of the enclosure. On the other hand, isotherms for $100 \leq \Lambda$ can be seen to be nearly parallel to either the lower or upper inclined surface except when close to the hot or cold surface, which is quite in contrast to the isotherms shown for the $\Lambda=10$ case. 

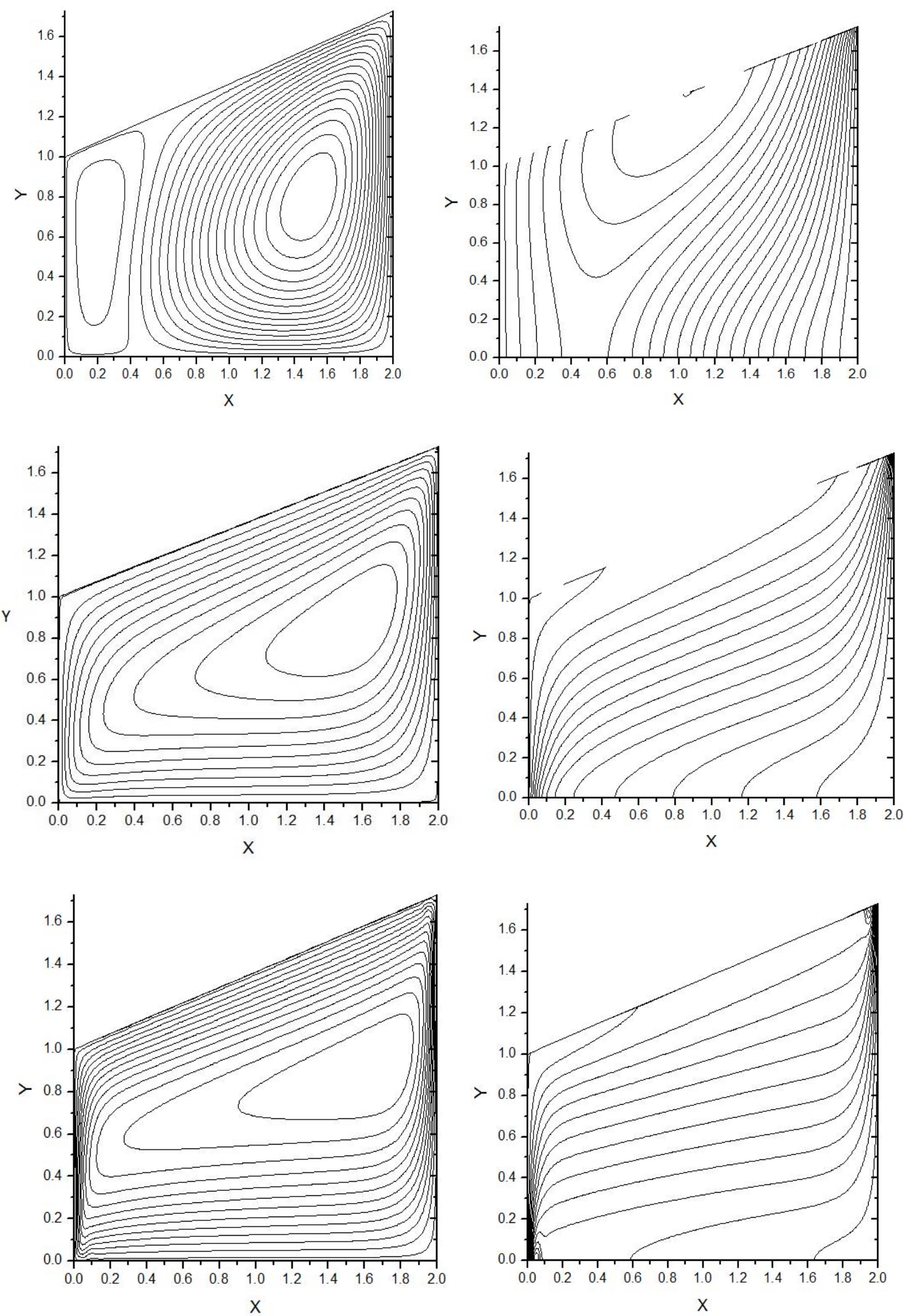

Fig.8. Streamlines (left) and isotherms (right) for $\theta=20^{\circ}, A r=2, Q=2$ : Top: $\Lambda=10$, Middle: $\Lambda=100$, Bottom: $\Lambda=500$. 

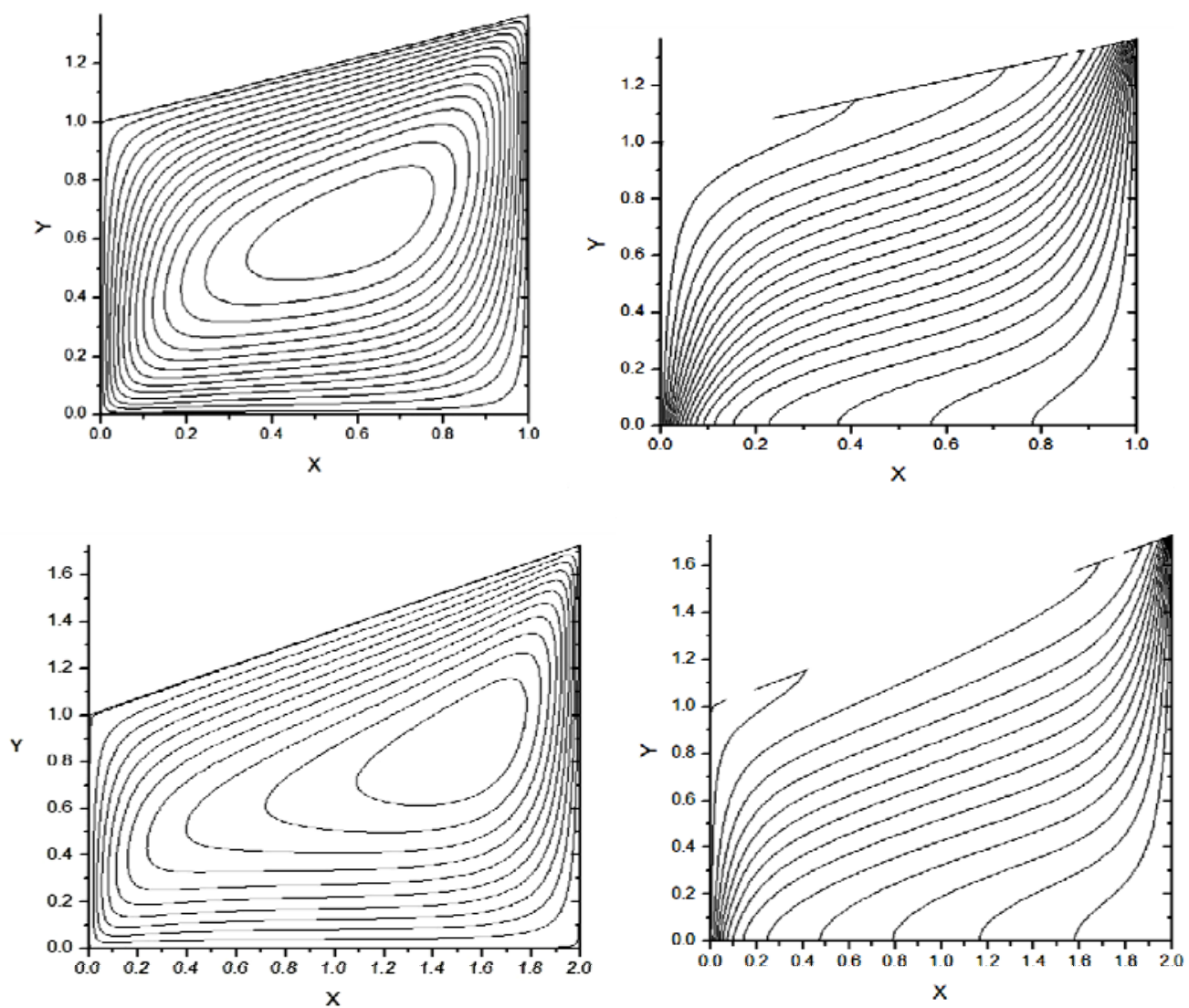

Fig.9. Streamlines (left) and isotherms (right) for $\theta=20^{\circ}, \Lambda=100, Q=2$ : Top: $A r=1$, Bottom: $A r=2$.
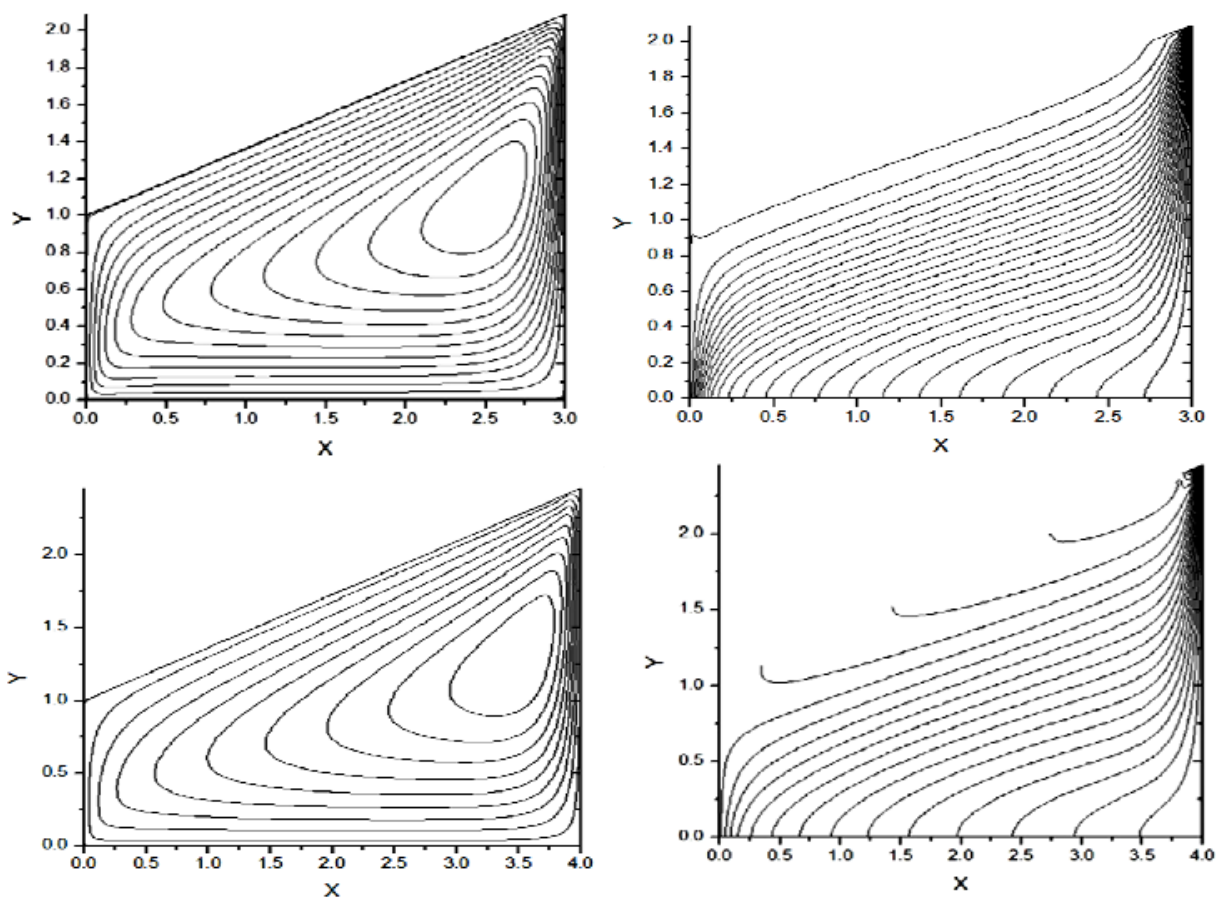

Fig.10. Streamlines (left) and isotherms (right) for $\theta=20^{\circ}, \Lambda=100, Q=2$ : Top: $A r=3$, Bottom: $A r=4$. 
The last set of Figs 9 and 10 shows how streamlines and isotherms respond to the variation in the aspect ratio $\operatorname{Ar}(=L / H)$ from 1 to 4 ; this is achieved by keeping $H$ fixed but letting $L$ assume values from $H$ to $4 H$. The streamline contours indeed show marked variation as $A r$ changes from 1 through 4. It can be seen that the uniform circulation pattern, nearly horizontal (Fig.9, top), undergoes significant changes in both shape and orientation as $A r$ increases beyond unity. The boundary layer effects too are seen to be concentrating on the right (cold) wall with enhancement of $A r$ values. On the examination of contours of isotherms in these figures, one observes a sort of transition as $A r$ increases beyond the value of 2. This change is apparent from the isotherm in Fig. 10 for $A r=3$ and $A r=4$. Even the pattern of the boundary layer region which corresponds to the region dominated by conduction, in comparison to convection currents, can be seen to undergo changes. For instance, the convection currents dominate all over the flow domain except for a very thin layer close to the cold wall, for higher values of $\mathrm{Ar}$.

Besides assessing the effect of governing parameters on isotherms and streamlines above, we have also computed values of the average Nusselt number on the warmer wall (see Tab.1) for two values of $\theta$ assuming $\Lambda$ and $A r$ to be fixed. In these tables, we have shown variation of the average Nusselt number with $\theta, K, \alpha$ and $Q$. In short, the effects of these parameters on $\mathrm{Nu}_{\mathrm{av}}$ can be summarized as follows:

As the parameter $Q$ increases from negative to positive values, the heat transfer rate at the wall undergoes a mild decrease. This indicates that less heat is transferred from the wall to the fluid in the presence of heat sinks. Physically, the opposite happens in the case of heat sources. This physical fact is clearly borne out by the values of the Nusselt number in Tab.1. The anisotropic effects of permeability and thermal diffusivity also have decreasing influence on the heat transfer; however, the transfer rates are much more pronounced for these processes. Finally, the heat transfer rate on the heated wall of the trapezoidal cavity is higher than that of a the rectangular one.

Table 1. Average Nusselt number for $A r=1, \Lambda=500$.

\begin{tabular}{|c|c|c|c|c|}
\hline$\theta$ & $K$ & $\alpha$ & $Q$ & $\mathrm{Nu}_{\mathrm{av}}$ \\
\hline 0 & 0.5 & 0.5 & -0.5 & 22.7018 \\
\hline 0 & 0.5 & 0.5 & 0.0 & 22.4618 \\
\hline 0 & 0.5 & 0.5 & 0.5 & 22.2189 \\
\hline 0 & 0.5 & 1.5 & -0.5 & 12.0823 \\
\hline 0 & 0.5 & 1.5 & 0.0 & 12.0167 \\
\hline 0 & 0.5 & 1.5 & 0.5 & 11.9507 \\
\hline 0 & 1.5 & 0.5 & -0.5 & 12.6709 \\
\hline 0 & 1.5 & 0.5 & 0.0 & 12.4311 \\
\hline 0 & 1.5 & 0.5 & 0.5 & 12.1864 \\
\hline 0 & 1.5 & 1.5 & -0.5 & 06.6985 \\
\hline 0 & 1.5 & 1.5 & 0.0 & 06.6287 \\
\hline 0 & 1.5 & 1.5 & 0.5 & 06.5581 \\
\hline 10 & 0.5 & 0.5 & -0.5 & 25.2008 \\
\hline 10 & 0.5 & 0.5 & 0.0 & 24.9690 \\
\hline 10 & 0.5 & 0.5 & 0.5 & 24.7344 \\
\hline 10 & 0.5 & 1.5 & -0.5 & 13.4243 \\
\hline 10 & 0.5 & 1.5 & 0.0 & 13.3608 \\
\hline 10 & 0.5 & 1.5 & 0.5 & 13.2970 \\
\hline 10 & 1.5 & 0.5 & -0.5 & 13.9202 \\
\hline 10 & 1.5 & 0.5 & 0.0 & 13.6877 \\
\hline 10 & 1.5 & 0.5 & 0.5 & 13.4503 \\
\hline 10 & 1.5 & 1.5 & -0.5 & 07.3601 \\
\hline 10 & 1.5 & 1.5 & 0.0 & 07.2925 \\
\hline 10 & 1.5 & 1.5 & 0.5 & 07.2243 \\
\hline
\end{tabular}




\section{Acknowledgement}

This work was supported by the Sultan Qaboos University Research Grant No. IG/SCI/DOMS/2014-10.

\section{Nomenclature}

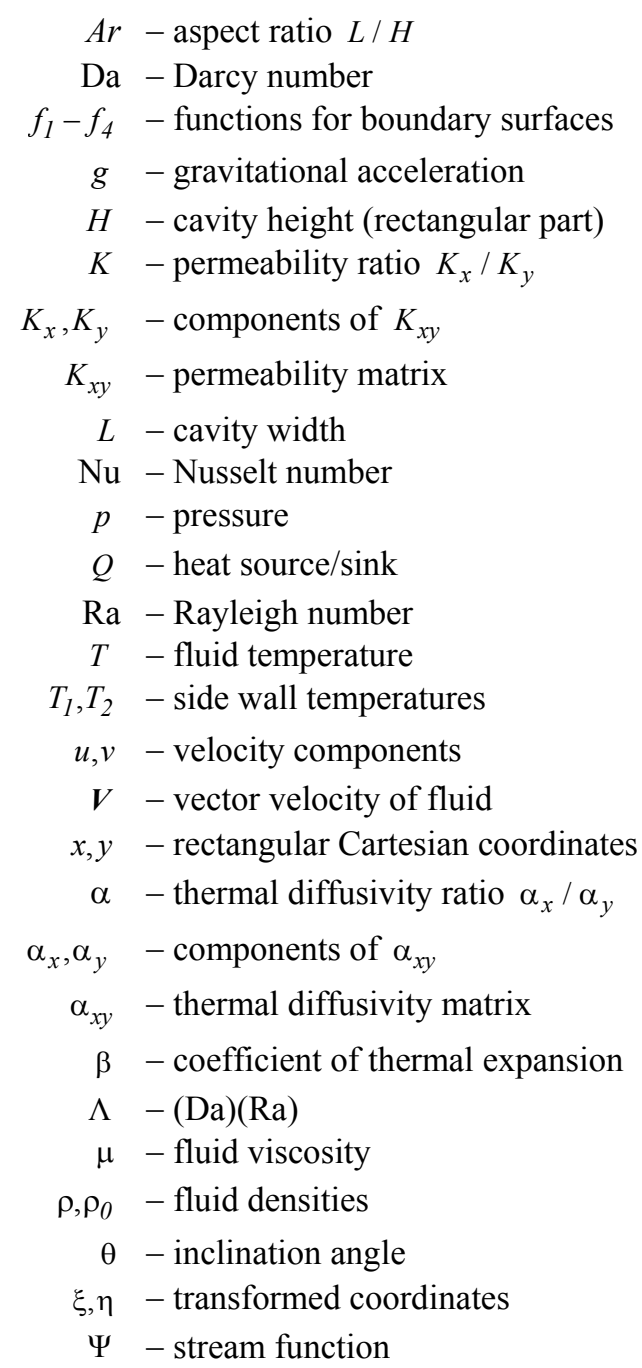

\section{References}

[1] Ni J. and Beckermann C. (1991): Natural convection in a vertical enclosure filled with anisotropic porous media. J. Heat Transfer, vol.113, pp.1033-1037.

[2] Chang W.J. and Liu H.C. (1994): Natural convection in a finite wall rectangular cavity filled with an anisotropic porous medium. - Int. J. Heat Mass Transfer, vol.37, pp.303-312.

[3] Kimura S., Masuda Y. and Hayashi T.K. (1993): Natural convection in an anisotropic porous medium heated from the side (effects of anisotropic properties of porous matrix). - Heat Transf. Jpn. Res, vol.22, pp.139-153.

[4] Tyvand P.A. and Storesletten L. (1991): Onset of convection in an anisotropic porous medium with oblique principal axes. - J. Fluid Mech, vol.226, pp.371-382.

[5] Storesletten L. (1993): Natural convection in a horizontal porous layer with anisotropic thermal diffusivity. Transp. Porous Media, vol.12, pp.19-29.

[6] Degan G., Vasseur P. and Bilgen E. (1995): Convective heat transfer in a vertical anisotropic porous layer. - Int. J. Heat Mass Transfer, vol.38, pp.1975-1987. 
[7] Degan G. and Vasseur P. (1996): Natural convection in a vertical slot filled with an anisotropic porous medium with oblique principal axes. - Numer. Heat Transfer-Part A, vol.30, pp.397-412.

[8] Mamou M., Mahidjida A., Vasseur P. and Robillard L. (1998): Onset of convection in an anisotropic porous medium heated from below by a constant heat flux. - Int. Comm. Heat Mass Transfer, vol.25, pp.799-808.

[9] Storesletten L. and Tveitereid M. (1999): Onset of convection in an inclined porous layer with anisotropic permeability. - Appl. Mech. Engng., vol.4, pp.575-587.

[10] Mahidjida A., Robillard L., Vasseur P. and Mamou M. (2000): Onset of convection in an anisotropic porous layer of finite lateral extent. - Int. Comm. Heat Mass Transfer, vol.27, pp.333-342.

[11] Nithiarasu P., Sujatha K.S., Ravindran K., Sundararajan T. and Seetharamu K.N. (2000): Non-Darcy natural convection in a hydrodynamically and thermally anisotropic porous medium. - Computer Meth. App. Mech. Engng, vol.188, pp.413-430.

[12] Malashetty M.S. and Basavaraja D. (2003): The effect of thermal/gravity modulation on the onset of convection in a horizontal anisotropic porous layer. - Int. J. Appl. Mech. Engng., vol.8, pp.425-439.

[13] Chandra P. and Satyamurty V.V. (2011): Non-Darcian and anisotropic effects on free convection in a porous enclosure. - Trans. Porous Media, vol.90, pp.301-320.

[14] Bhadauria B.S., Srivastava A.K., Sacheti N.C. and Chandran P. (2012): Gravity modulation of thermal instability in a viscoelastic fluid saturated anisotropic porous medium. - Zeit. Naturforschung, vol.67, pp.1-9.

[15] Tiwari A.K., Singh A.K., Chandran P. and Sacheti N.C. (2012): Natural convection in a cavity with a sloping upper surface filled with an anisotropic porous material. - Acta Mech., vol.223, pp.95-108.

[16] Bhadauria B.S. and Kiran P. (2013): Heat transport in an anisotropic porous medium saturated with variable viscosity liquid under temperature modulation. - Trans. Porous Media, vol.100, pp.279-295.

[17] Jha B.K. (2001): Transient free-convective flow in a vertical channel with heat sinks. - Int. J. Appl. Mech. Engng, vol.6, pp.279-286.

[18] Degan G., Vasseur P. and Awanou N.C. (2005): Anisotropy effects on non-Darcy natural convection from concentrated heat sources in porous media. - Acta Mech., vol.179, pp.111-124.

[19] Jaya Krishna D., Basak T. and Das S.K.: Natural convection in a heat generating hydrodynamically and thermally anisotropic non-Darcy porous medium. - Int. J. Heat Mass Transfer, vol.51, pp.4691-4703.

[20] Bhadauria B.S., Kumar A., Kumar J., Sacheti N.C. and Chandran P. (2011): Natural convection in a rotating anisotropic porous layer with internal heat. - Trans. Porous Media, vol.90, pp.687-705.

[21] Bhadauria B.S., Hashim I. and Siddheshwar P.G.: Study of heat transport in a porous medium under G-jitter and internal heating effects. - Trans. Porous Media, vol.96, pp.21-37.

[22] Vanishree R.K. (2014): Effects of throughflow and internal heat generation on a thermo-convective instability in an anisotropic porous medium. - J. Appl. Fluid Mech., vol.7, pp.581-590.

[23] Bhadauria B.S., Singh M.K., Singh A., Singh B.K. and Kiran P. (2016): Stability analysis and internal heating effects on oscillatory convection in a viscoelastic fluid saturated porous medium under gravitation modulations. Int. J. Appl. Mech. Engng., vol.21, pp.785-803.

[24] Moretti G. and Abbett M. (1966): A time-dependent computational method for blunt body flows. - AIAA J, vol.4, pp.2136-2141.

[25] Mallinson G. and de Vahl Davis G. (1973): The method of the false transient for the solution of coupled elliptic equations. - J. Comput. Phys., vol.12, pp.435-461.

[26] Smith R.E. (1982): Algebraic grid generation. - In: Numerical Grid Generation (J.F. Thompson, Ed.).Amsterdam: North Holland.

[27] Shih T.I., Bailey R.T., Nguyen H.L. and Roelke R.J. (1991): Algebraic grid generation for complex geometries. Int. J. Num. Meth. Fluids, vol.13, pp.1-31. 